\title{
Horizontal lateral lamella as a risk factor for iatrogenic cerebrospinal fluid leak. Clinical retrospective evaluation of 24 cases* $^{*}$
}

\author{
A. Preti',2, F. Mozzanica', R. Gera', S. Gallo², J. Zocchi², F. Bandi², G. Guidugli', \\ F. Ambrogi', A. Yakirevitch ${ }^{3}$, A. Schindler ${ }^{4}$, A. Dragonetti ${ }^{5}$, P. Castelnuovo ${ }^{6}$, \\ F. Ottaviani ${ }^{1}$ \\ ' Department of Biomedical and Clinical Sciences, University of Milan, Milan, Italy \\ 2 Department of Biotechnology and Life Sciences, University of Insubria, Varese, Italy \\ ${ }^{3}$ Department of Otorhinolaryngology - Head and Neck Surgery, Sheba Medical Center and Sackler School of Medicine, Tel Aviv \\ University, Israel \\ ${ }^{4}$ Department of Biochemical and Clinical science "Luigi Sacco", University of Milan, Milan, Italy \\ ${ }^{5}$ Department of Otolaryngology, Ospedale Niguarda, Milan, Italy \\ ${ }^{6}$ Unit of Otorhinolaryngology, Department of Biotechnology and Life Sciences, University of Insubria, Varese, Italy
}

Rhinology 56: 4, 358-363, 2018

https://doi.org/10.4193/Rhin.18.013

*Received for publication:

January 17,2018

Accepted: March 18, 2018

Background: Several authors highlighted the limitations of the Keros classification system in predicting intracranial entry risk. Recently, our group proposed a new classification system based on the angle formed between the lateral lamella of the cribriform plate (LLCP) and the continuation of an horizontal plane passing through the cribriform plate (Gera classification). The aim of this study was to analyze whether the risk of iatrogenic cerebrospinal fluid leak (CSF-L) was better predicted by Keros or Gera classification.

Methodology: The pre-operative CT scans of 24 patients (CSF-L group) who suffered from iatrogenic CSF-L during endoscopic sinus surgery (ESS) were compared to those obtained from a group of 100 patients who underwent uneventful ESS (control group). The skull base measurements as well as the distribution of Keros and Gera classes in the 2 groups were analyzed.

Results: No difference in the distribution of Keros classes or in the depth of the cribriform plate between CSF-L and control group were demonstrated. On the contrary, significant differences in the distribution of Gera classes and in the degree of the angle formed by the LLCP and the continuation of the horizontal plane passing through the cribriform plate were found. In particular, according to Gera classification system, 19 out of 24 patients in the CSF-L group were considered at risk for iatrogenic CSF-L.

Conclusions: Gera classification system might be more sensitive to anatomical variations associated with CSF-L than the Keros' one, further suggesting the application of the former during the preoperative CT scan evaluation.

Key words: CSF leak, radiology, surgery, anatomy, skull base

\section{Introduction}

Increasing of the surgical expertise and technological advances in instrumentations, as well as a deeper anatomical knowledge, have significantly reduced the rate of complications during endoscopic sinus surgery (ESS). Nonetheless, cerebrospinal fluid leak (CSF-L) still represents a possible complication of ESS(1). In the majority of the cases, CSF-L occur during ethmoidectomy and might be related to anatomical variations of the anterior skull base ${ }^{(2,3)}$, here composed by the cribriform plate and by the fovea ethmoidalis (the most anterosuperior part of the anterior ethmoidal roof included between the anterior ethmoidal artery and the posterior border of the frontal recess). These structures converge at the lateral lamella of the cribriform plate (LLCP). The latter delimits laterally the cribriform groove and represents the thinnest and the most vulnerable structure of the anterior skull base as its thickness can be only $0.05 \mathrm{~mm}^{(2,4)}$. For this reason, the LLCP is the most frequent site of iatrogenic skull base injury due to direct penetration or fracture during middle turbinate 
manipulation or lack of awareness of instrument placement ${ }^{(4)}$. If not promptly identified and treated this complication can lead to serious neurologic sequelae ${ }^{(5)}$.

Several authors suggested that the risk of iatrogenic LLCP injury increases with the olfactory cleft depth ${ }^{(6-7)}$. For this reason, the classification proposed in 1962 by $\operatorname{Keros}^{(8)}$ is still the most commonly used today to estimate the risk of iatrogenic CSF-L. It distinguishes between 3 types of cribriform fossa depending on its depth measured on computed tomography (CT) images: type 1, 1-3 mm, type 2, 4-7 $\mathrm{mm}$ and type 3, more than $7 \mathrm{~mm}$. Patients with Keros type 3 are supposed to have a greater risk of intracranial entry since the more pronounced depth of the cribriform cleft and the thinness of its bony wall make this area more susceptible to iatrogenic injuries ${ }^{(2,7)}$. Even though widely used, several authors highlighted the limitations of this classification system in fully describing the shape of the skull base and predicting the intracranial entry risk ${ }^{(6,9-13)}$. Recently, our group proposed a new classification system of the anterior skull base anatomy based on the angle formed between the LLCP and the lateral extension of an horizontal plane passing through the cribriform plate (Gera classification). In particular, the angle was classified into class I ( $>80$ degrees, low risk), class II (45 to 80 degrees, medium risk), and class III ( $<45$ degrees, high risk) according to the theoretical risk of iatrogenic injuries ${ }^{(2)}$. We have previously observed that the angulation of the LLCP, by affecting the height of the ethmoidal roof relative to the cribriform plate ${ }^{(14)}$, can be a major risk factor of iatrogenic injuries during the dissection of the anterior ethmoidal cells and the frontal recess. During these surgical steps the instruments are in close proximity to the LLCP, thus it is possible that a more pronounced ethmoidal roof slope may predispose to inadvertent skull base violation ${ }^{(2)}$.

In order to evaluate the clinical implications of Gera classification system, we retrospectively analyzed the preoperative CT scans of patients with chronic rhinosinusitis (CRS) who suffered from intraoperative iatrogenic CSF-L. The underlying hypothesis is that patients who experienced an intraoperative CSF-L would fall more frequently into class III (high risk of iatrogenic injuries) according to Gera classification. The importance of this study lies in the fact that the availability of a classification system able to recognize the anatomical variations predisposing to iatrogenic CSF-L may allow a more accurate interpretation of the preoperative CT findings and, thus, be of further help in preventing major surgical complications.

\section{Materials and methods}

In this retrospective study the iatrogenic complications of a total of 6023 ESS procedures for CRS, performed in 4 different rhinologic centers in the period between January 2012 and January 2016, were analyzed. The surgeries were performed by 6 surgeons with an experience of more than 10 years. Among the

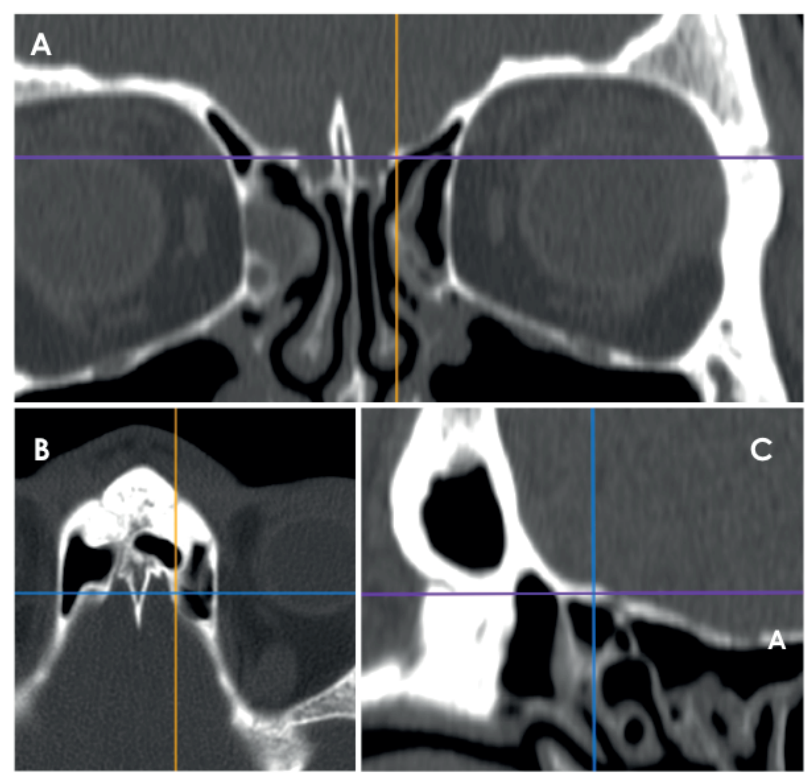

Figure 1. Point of assessment of the measurements taken: the convergence between the fovea ethmoidalis and the LLCP on the frontal plane (A) at the level of the anterior wall of the first fovea ethmoidalis on the sagittal plane (C). Correspondence of the point in the axial plane (B).

6023 patients who underwent ESS, 24 suffered from iatrogenic CSF-L due to LLCP injury. The study was conducted according to the declaration of Helsinki and was previously approved by the Institutional Review Boards of the enrolled hospitals.

The preoperative sinus CT scans of the patients with CSF-L were evaluated (CSF-L group). In addition, a total of 100 preoperative sinus CT scans ( 25 for each of the enrolled rhinologic centers) of randomly selected CRS patients who underwent uneventful ESS (performed by the same surgeons in the same period) were also evaluated (control group).

Patients younger than 18 years, with previous trauma, sinonasal tumor, congenital facial malformations or skull base defects were excluded. In addition, none of the patients had been previously treated with any kind of sinonasal surgery.

\section{CT measurements}

All CT scans were performed on high-speed spiral CT scanners using non-contrast axial $1.5-\mathrm{mm}$ sections. Scans that deviated from a true coronal plane by more than $5^{\circ}$ from the perpendicular plane of the floor of the nose were also excluded. The morphological evaluation was performed on high-resolution coronal and sagittal sections using a specialized computer software, the Picture Archiving and Communication System (PACS) ${ }^{(15)}$. Similarly to our previous study ${ }^{(2)}$ two skull base measurements were assessed. Both were taken, using a 3-D multi-planar reconstruction (MPR), at the convergence between the LLCP and the most antero-superior part of the anterior ethmoidal roof $^{(16)}$ on the coronal plane and at the level of the posterior 


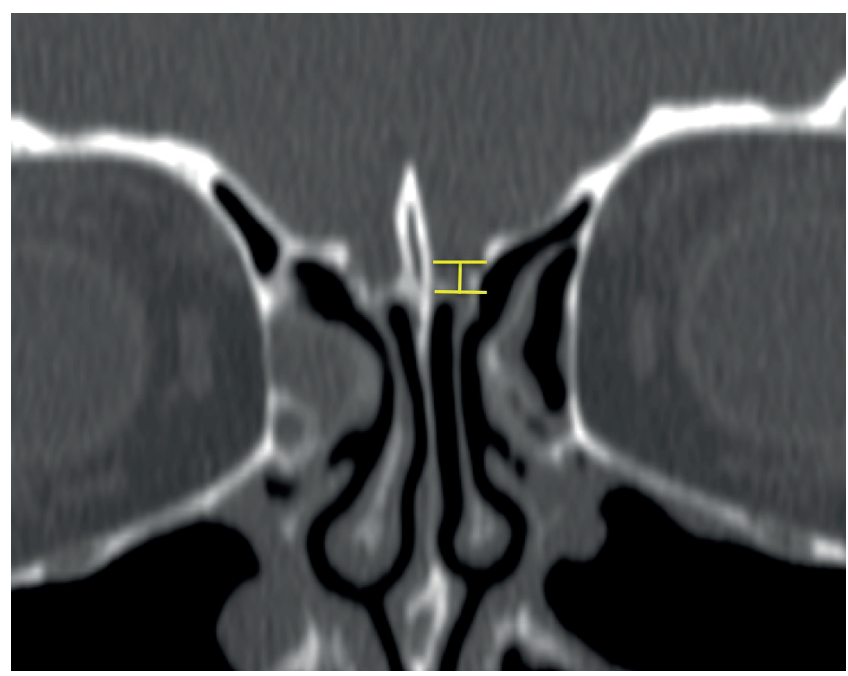

Figure 2. Depth of the cribriform plate: measured as the vertical height of the olfactory fossa in the coronal plane.

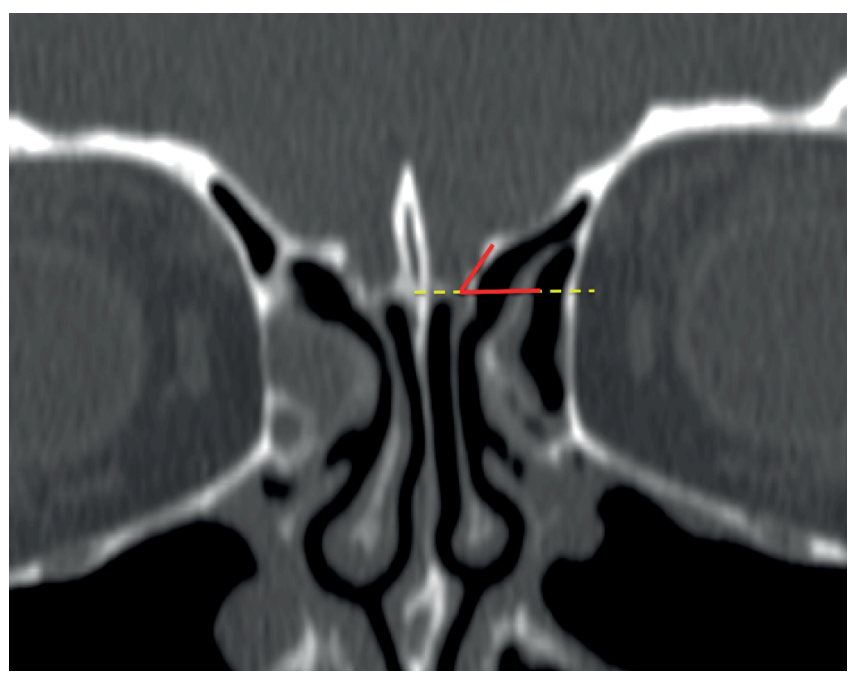

Figure 3. Angle between the LLCP and continuation of the horizontal plane passing through the cribriform plate (treaty line), defining the Gera's classification. margin of frontal recess on the sagittal plane (Figure 1). This point was selected because it is well identifiable and represents an important landmark for ethmoidal dissection and during the approaches to the frontal sinus. In addition, our previous study demonstrated that both intra- and inter-rater reliability for measurements taken in this point were high, suggesting a good reproducibility ${ }^{(2)}$.

In particular, we measured the:

- Depth of the cribriform plate: measured as the vertical height of the olfactory cleft in the coronal plane on each side (see Figure 2). The depth was defined as type 1 (1-3 $\mathrm{mm}$ depth), type 2 (4-7 $\mathrm{mm}$ depth) or type 3 (more than 7 $\mathrm{mm}$ depth), according to Keros classification system.

- Degree of the angle formed by the LLCP and the continuation of the horizontal plane passing through the cribriform plate (see Figure 3). According to Gera system ${ }^{(2)}$, the angle was classified depending on its degree and on the hypothetical risk of iatrogenic injuries as class I (> 80 degrees, low risk), class II (45 to 80 degrees, medium risk) or class III ( $<45$ degrees, high risk).

\section{Statistical analysis}

The results of skull base measurements obtained in the CSF-L group were analyzed using Mann-Whitney and Fisher test (for continuous and categorical data respectively) in order to evaluate the presence of significant differences related to the side of CSF-L. In addition, the results obtained in the CSF-L group were compared to those obtained in the control group using the Fisher and Mann-Whitney tests when appropriate. Finally, the sensitivity, specificity, positive and negative predictive value for both Keros and Gera classification systems in the identifica- tion of patients at risk for developing intraoperative CSF-L were assessed. For this purpose, the classification systems were dichotomized in order to identify patients at high risk (class III for Gera and type 3 for Keros classification system, respectively) and at lower risk (class I and II for Gera and type 1 and 2 for Keros classification system, respectively).

\section{Results}

The percentage of iatrogenic CSF-L at the level of the LLCP in our sample was $0,398 \%$. A total of 124 preoperative sinus CT scans ( 24 for the CSF-L group and 100 for the control one) were retrospectively analyzed. The mean age of patients in CSF-L group was $53.5 \pm 6.9$ years (range 25-74) and $50.8 \pm 7.1$ (range 21-71) in the control group. The difference was not significant on Mann-Whitney test $(p=0.387)$. Male to female ratio was $16: 8$ in the CSF-L group and 73:27 in the control group without significant difference between the two groups according to Fisher test $(p=0.532)$. Right and left iatrogenic injuries occurred equally in 12 cases on each side. There were no cases of bilateral CSF-L. Crista galli was perpendicular to the midline in all the patients in CSF-L group. Data on skull base measurements obtained in CSF$\mathrm{L}$ and control group are summarized in Table 1 and 2 .

\section{Depth of the cribriform plate}

In the CSF-L group the mean depth of the cribriform plate was $4.3 \pm 1.7 \mathrm{~mm}$ at CSF-L side and $4.5 \pm 1.6 \mathrm{~mm}$ at the contralateral one. This difference was found not significant on Mann-Whitney test $(p=0.802)$. The most common anatomical variation was Keros type 2 both in CSF-L and contralateral side (13 patients, $54.2 \%$, and 15 patients, $62.5 \%$ respectively). These differences were found not significant on Fisher test $(p=0.545)$. 
Table 1. Distribution of skull base measurements in the two groups of patients. The mean \pm standard deviation are reported as well as the results of comparison between the parameters obtained in the CSF-L and control groups through Mann Whitney test. Ranges are reported in brackets. Angle = degree of the angle formed by the LLCP and continuation of the horizontal plane passing through the cribriform plate.

\begin{tabular}{lccccccc} 
& & CSF-L group $(\mathbf{n}=\mathbf{2 4})$ & & \multicolumn{3}{c}{ Control group $(\mathbf{n}=100)$} \\
& CSF-L side & Controlateral & p & Right & Left & P \\
\hline Depth of the cribriform & $4.3 \pm 1.7$ & $4.5 \pm 1.6$ & 0.802 & $5.2 \pm 1.7$ & $5.5 \pm 1.6$ & 0.839 \\
plate $(\mathrm{mm})$ & $(2.3-8.0)$ & $(2.1-6.8)$ & & $(2.4-10.1)$ & $(2.8-10.3)$ & \\
Angle (degrees) & $41.2 \pm 10.3$ & $50.1 \pm 10.4$ & 0.003 & $71.7 \pm 12.4$ & $71.1 \pm 11.2$ & 0.799 \\
& $(27-48)$ & $(30-65)$ & & $(30-88)$ & $(28-90)$ &
\end{tabular}

Table 2: Distribution of Keros and Gera classes in the two groups of patients. The results of the Fisher test are also reported. Percentages are reported in brackets.

\begin{tabular}{|c|c|c|c|c|c|c|c|}
\hline \multicolumn{2}{|c|}{ Classes } & \multicolumn{3}{|c|}{ CSF-L group $(n=24)$} & \multicolumn{3}{|c|}{ Control group $(n=100)$} \\
\hline & & CSF-L side & Controlateral & $\mathbf{p}$ & Right & Left & $\mathbf{p}$ \\
\hline \multirow[t]{3}{*}{ Keros } & 1 & $10(41.7 \%)$ & $9(37.5 \%)$ & 0.545 & $23(23 \%)$ & $30(30 \%)$ & 0.497 \\
\hline & 2 & $13(54.2 \%)$ & $15(62.5 \%)$ & & $61(61 \%)$ & 59 (59\%) & \\
\hline & 3 & $1(4.1 \%)$ & $0(0 \%)$ & & $16(16 \%)$ & $11(11 \%)$ & \\
\hline \multirow[t]{3}{*}{ Gera class } & I & $1(4.1 \%)$ & $0(0 \%)$ & 0.005 & $32(32 \%)$ & $31(31 \%)$ & 0.887 \\
\hline & ॥ & $4(16.7 \%)$ & $14(58.3 \%)$ & & $64(64 \%)$ & $65(65 \%)$ & \\
\hline & III & 19 (79.2\%) & 10 (41.7\%) & & $4(4 \%)$ & $4(4 \%)$ & \\
\hline
\end{tabular}

Table 3. Sensitivity, specificity, positive predictive value (PPV) and negative predictive value (NPV) for Keros and Gera classification system. Confidence intervals at $90 \%$ are reported in brackets.

\begin{tabular}{|lcccc|} 
& Sensitivity & Specificity & PPV & NPV \\
\hline Keros classification & $4.2 \%(1.6 \%-9.8 \%)$ & $84 \%(76.1 \%-89.7 \%)$ & $5.9 \%(2.7 \%-12 \%)$ & $78.5 \%(70 \%-85.2 \%)$ \\
Gera classification & $79.2 \%(70.8 \%-85.7 \%)$ & $96 \%(90.4 \%-98.5 \%)$ & $82.6 \%(74.5 \%-88.6 \%)$ & $95 \%(89.2 \%-97.9 \%)$ \\
\hline
\end{tabular}

In the control group the mean depth of the cribriform plate was $5.2 \pm 1.7 \mathrm{~mm}$ at the right side and $5.5 \pm 1.6 \mathrm{~mm}$ at the left side. This difference was found not significant on Mann-Whitney test $(p=0.839)$. The most common anatomical variation both in the right and left side was Keros type 2 ( 61 patients, $61 \%$, and 59 patients, $59 \%$ respectively). These differences were found not significant on Fisher test $(p=0.497)$.

No significant differences in the depth of the cribriform or in the distribution of Keros classes were demonstrated at MannWhitney and Fisher tests $(p=0.451$ and $p=0.243$ respectively) between patients in CSF-L and control groups.

\section{Degree of the angle of LLCP}

In the CSF-L group the mean degree of the angle formed by the LLCP and the continuation of the horizontal plane passing through the cribriform plate was $41.2 \pm 10.3^{\circ}$ in the CSF-L side, while it was $50.1 \pm 10.4^{\circ}$ in the contralateral one. This difference was found significant on Mann-Whitney test $(p=0.003)$. According to Gera classification system ${ }^{(2)}$, the most common anato- mical variation in the CSF-L side was angle class III (19 patients, 79.2\%). In the contralateral side the most common anatomical variation was angle class II (14 patients, 58.3\%). These differences were found significant on Fisher test $(p=0.005)$.

In the control group the mean degree of the angle was $71.7 \pm$ $12.4^{\circ}$ in the right side and $71.1 \pm 11.2^{\circ}$ in the left side. These differences were found not significant on Mann-Whitney test $(p=$ 0.799). The most common anatomical variation was angle class II both in the right and left side ( 64 patients, $64 \%$, and 65 patients, $65 \%$ respectively). These differences were found not significant on Fisher test $(p=0.887)$.

Significant differences were demonstrated both in the mean degree of the angle ( $p=0.002$ at Mann-Whitney test), and in the distribution of angle classes according to Gera classification ( $p=0.010$ at Fisher test) between patients in CSF-L and control group. In particular, patients in CSF-L group scored lower in the mean degree of the angle and were more frequently classified as angle class III. 
The sensitivity, specificity, positive and negative predictive values for Keros and Gera classification systems are reported in Table 3. Gera classification system scored higher in all the above mentioned parameters.

\section{Discussion}

In this retrospective study, the CT scans of 124 patients who underwent ESS for CRS were analyzed according to Gera and Keros classifications in order to evaluate which is the better indicator for a high risk of iatrogenic CSF-L. The underlying hypothesis is that a more pronounced slope of the anterior skull base on the coronal plane may predispose to inadvertent skull base violation during the dissection of the more anterior ethmoidal cells and during the approach to the frontal sinus. For this reason, we speculated that the Gera classification system could be more informative than the Keros' one regarding the anatomical variations more frequently associated to iatrogenic CSFL.

The results here reported appear promising and further suggest the utility of Gera classification combined with the Keros' one during the preoperative CT assessment in order to prevent major complications. In our sample, the distribution of Keros classes in the control group appeared similar to those previously reported. The most common anatomical variation was Keros type 2 (61\%), followed by type 1 (23\%), and type 3 (16\%). In our previous study $^{(2)}$ on 190 CT scans, the results were almost equal. The mean depth of cribriform plate found in the control group appeared similar to those previously reported. Guler et al. ${ }^{(17)}$ found a mean height of the ethmoidal roof ranging from 3.9 $\mathrm{mm}$ to $4.5 \mathrm{~mm}$ at the right side and from $3.9 \mathrm{~mm}$ to $4.4 \mathrm{~mm}$ at the left side. Erdem et al. ${ }^{(18)}$ found a mean height of the ethmoidal roof of $6.1 \mathrm{~mm}$ at the right side and $6.3 \mathrm{~mm}$ at the left side. Meloni et al. ${ }^{(19)}$ defined the mean depth of the cribriform as 5.9 $\mathrm{mm}$ in 106 Italian patients. Concerning the distribution of Gera classes in the control group, our results appear very similar to those previously reported. In particular, also in our previous study ${ }^{(2)}$ the vast majority of patients were classified as class I and II, while only $4.6 \%$ of them were classified as at high risk for iatrogenic injuries.

Similar to our control group, in the CSF-L group the most common anatomical variation was Keros type 2, followed by Keros type 1, both at side of the CSF-L and at the contralateral one. Only 1 patient was classified as at high risk (Keros type 3). No differences in the distribution of Keros classes or in depth of the cribriform plate between CSF-L and control group were demonstrated. On the contrary, according to Gera classification system ${ }^{(2)}$, the most common anatomical variation at the CSF-L side was angle class III (19 out of 24 patients). The difference in the mean angle degree and in the distribution of Gera classes among patients in control and CSF-L groups was found significant, as well as the difference in the mean angle degree and in the Gera classes distribution between CSF-L side and contrala- teral one in the CSF-L group. These findings are similar to those reported by Heaton et al. ${ }^{(10)}$ who compared the slope of the skull base on the coronal plane in CSF-L patients and in a control group and concluded that CSF-L patients have a larger slope of the skull base than those of the control group.

The data here reported suggest that CSF-L patients were characterized by a lower angle degree. In addition, the degree of the angle was significantly lower at the side of the CSF-L. It is consequently possible to speculate that a classification system based on the degree of the angle (such as the Gera's one) might be more sensitive to anatomical variations associated with CSF-L than that of Keros. According to the latter, only 1 out of 24 patients was considered at high risk to develop an iatrogenic injury. On the other hand, according to Gera classification 19 out of 24 patients were considered at high risk. The higher values of sensitivity, specificity, PPV and NPV for the Gera classification system compared to the Keros' one, further suggest the application of the former during the preoperative CT scan evaluation. However, this consideration must be taken with caution. The Keros classification, in fact, is well known and widely used during the pre-operative assessment of CT scans. It is consequently possible that surgeon might have paid more attention during ESS procedures in patients with Keros type 3. This could have reduced the CSF-L rate associated to Keros type 3 in our sample. For this reason, only a prospective study would be able to proof that Gera classification could be considered a better indicator for a high risk of CSF-L.

This study presents some limitations that are related (a) to the small number of patients with CSF-L, and (b) to the multiple centers involved in the case collection.

a. We could argue that the small number of patients is related to low rate of CSF-L occurring during ESS. In particular, the percentage of CSF-L among the 6023 procedures was $0.398 \%$. This datum is in accordance with those previously reported. Hosemann et al. ${ }^{(20)}$ reported a CSF-L rate of $0.2-0.8 \%$ during ESS. In order to collect a sufficient number of patients with CSF-L for group comparison we were consequently forced to collect data from different centers. Even if enlarging the sample is surely useful from a statistical point of view, it adds some variables in the analysis (study limitation b).

b. The four rhinologic centers involved in the study have high volume of ESS procedures. Yet, each facility has its own expertise and a total of 6 surgeons performed the surgical procedures. This aspect could influence the results since it was previously reported that less experienced surgeons have a higher risk of injuring the dura, while more experienced surgeons performing increasingly extensive surgery could cause dramatic complications with medico-legal consequences ${ }^{(20)}$.

\section{Conclusion}

In conclusion, intraoperative iatrogenic CSF-L still represents a 
possible complication during ESS procedures that even a large surgical experience can not prevent. Despite the advances in the technological devices, instrumentations and surgical techniques, a deep anatomical knowledge and a meticulous preoperative analysis of the imaging remain a keystone in preventing this complication. Gera classification system presents good sensitivity and specificity in predicting a possible LLCP injury during ESS and its application, together with the Keros one, may be useful in the preoperative CT scan evaluation.

\section{Acknowledgement}

Andrea Preti is a PhD student of the "Experimental and Translational Medicine" course at University of Insubria.

Stefania Gallo is a PhD student of the "Biotechnology, Biosciences and Surgical Technology" course at University of Insubria.

\section{Authorship contribution}

FO, PC, RG, AS, AD, AY: Substantial contributions to the conception or design of the work, revising it critically for important intellectual content, final approval of the version to be published. SG, JZ, FB, GG: Substantial contributions to the acquisition of data for the work, revising it critically for important intellectual content and final approval of the version to be published. AP, FM: Substantial contributions to the analysis and interpretation of data for the work. Drafting the work. Final approval of the version to be published. FA, FM: Substantial contributions to the statistical analysis and the interpretation of data for the work, revising it critically for important intellectual content and final approval of the version to be published.

\section{Conflict of interest}

Each author has no conflict of interest to declare. study.

\section{References}

1. Ulualp SO. Complications of endoscopic sinus surgery: appropriate management of complications. Curr Opin Otolaryngol Head Neck Surg 2008; 16: 252-259.

2. Gera R, Mozzanica F, Karligkiotis A, et al. Lateral lamella of the cribriform plate, a keystone landmark: proposal for a nove classification system. Rhinology 2017; 25. [Epub ahead of print]

3. Dessi P, Castro F, Triglia JM, et al. Major complications of the sinus surgery: a review of 1192 procedures. J Laryngol Otol 1985; 108: 212-215.

4. Ohnishi T, Yanigasawa E. Lateral lamella of the cribriform plate an important high-risk area in endoscopic sinus surgery. Ear Nose Throat J 1995; 74; 688-690.

5. Kubik M, Lee S, Snyderman C, Wang E. Neurologic sequelae associated with delayed identification of iatrogenic skull base injury during endoscopic sinus surgery (ESS). Rhinology 2017; 55: 53-58.

6. Skorek A, Tretiakow D, Szmuda T, Przewozny T. Is the Keros classification alone enough to identify patients with the 'dangerous ethmoid'? An anatomical study. Acta OtoLaryngologica 2017; 137: 2.

7. Kainz J, Stammberger $H$. The roof of the anterior ethmoid: a locus minoris resistentiae in the skull base. Laryngorhinootologie 1988; 66; 142-149.

8. Keros P. On the pratical value of differences in the level of the lamina cribrosa of the ethmoid. Laryngorhinootologie 1962; 41: 808-813.

9. Kainz J, Stammberger $\mathrm{H}$. The roof of the anterior ethmoid: a locus minoris resistentiae in the skull base. Laryngorhinootologie 1988; 66; 142-149.

10. Heaton CM, Goldberg AN, Pletcher SD Glastonbury CM. Sinus Anatomy Associated With Inadvertent Cerebrospinal Fluid Leak During Functional Endoscopic Sinus Surgery. Laryngoscope 2012; 122: 1446 1449

11. Ramakrishnan VR, Suh JD, Kennedy DW Ethmoid skull-base height: a clinically relevant method of evaluation. Int Forum Allergy Rhinology 2011; 11: 396-400.

12. Lee JC, Song YJ, Chung YS, et al. Height and shape of the skull base as risk factors for skull base penetration during endoscopic sinus surgery. Ann Otol Rhinol Laryngol 2007; 116: 199-205.

13. Lebowitz RA, Terk A, Jacobs JB, et al. Asymmetry of the ethmoid roof: analysis using coronal computed tomography. Laryngoscope 2001; 111: 2122-2124.

14. Meyers RM, Valvassori G. Interpretation of anatomic variations of computed tomography scans of the sinuses: a surgeon's perspective. Laryngoscope 1998; 108: 422-425.

15. Savvateeva DM, Guldner C, Murthum T, et al. Digital volume tomography (DVT) measurements of the olfactory cleft and olfactory fossa. Acta Oto-Laryngologica 2010; 130: 398-404

16. Lund VJ, Stammberger H, Fokkens WJ, et al. European position paper on the anatomical terminology of the internal nose and paranasal sinuses. Rhinol Suppl 2014; 24: 1-34.

17. Guler C, Uysal IO, Polat K, et al. Analysis of the ethmoid roof and skull base with coronal section paranasal sinus computed tomography. J Craniof Surg 2012; 23: $1460-$ 1464

18. Erdem G1, Erdem T, Miman MC, Ozturan O. A radiological anatomic study of the cribriform plate compared with constant structures. Rhinology 2004; 42: 225-229.

19. Meloni F, Mini R, Rovasio S, et al. Anatomic variations of surgical importance in ethmoid labyrinth and sphenoid sinus. A study of radiologic anatomy. Surg Radiol Anat 1992: 14: 65-70.

20. Hosemann W, Draf C. Danger points, complications and medico-legal aspects in endoscopic sinus surgery. GMS Curr Top Otorhinolaryngol Head Neck Surg 2013; 12: Doc06.

Francesco Mozzanica, MD

Department of Biomedical and Clinical Sciences

University of Milan

Via G.B. Grassi 74

Milan

Italy

Tel: +39023903526

E-mail:

Francesco.mozzanica@gmail.com 